\title{
On Homogeneously Representable Interval Graphs
}

\author{
Marcin Krzywkowski · Jerzy Topp
}

Received: 2 June 2014/Revised: 1 October 2014/ Accepted: 13 January 2015 / Published online: 20 November 2015

(C) The Author(s) 2015. This article is published with open access at Springerlink.com

\begin{abstract}
We determine all graphs whose line graphs (middle graphs, total graphs, respectively) are homogeneously representable interval graphs.
\end{abstract}

Keywords Line graph · Middle graph · Total graph · Interval graph

A graph $G=(V, E)$ is said to be an interval graph if it is possible to assign to each vertex of $G$ a closed interval on the real line such that two distinct vertices of $G$ are adjacent if and only if the corresponding intervals have a non-empty intersection, that is, if there exists a collection $\mathcal{I}=\left\{I_{v} v \in\right.$ $V(G)\}$ of closed intervals on the real line such that $G$ is isomorphic to the intersection graph $\Omega(\mathcal{I})$ of $\mathcal{I}$. In such a situation, the collection $\mathcal{I}$ is called an interval representation of $G$. Without loss of generality we may assume that an interval representation consists of closed, nonempty, finite intervals in which all end points of the intervals are distinct. The first characterization of interval graphs has been proved by Lekkerkerker and Boland [1]. In some applications of interval graphs it is desirable to have an interval graph with

M. Krzywkowski

Department of Mathematics, University of Johannesburg,

Johannesburg, South Africa

M. Krzywkowski $(\bowtie)$

Faculty of Electronics, Telecommunications and Informatics,

Gdańsk University of Technology, Gdańsk, Poland

e-mail: marcin.krzywkowski@gmail.com

J. Topp

Institute of Informatics, University of Gdańsk, Gdańsk, Poland

e-mail: jerzy.topp@inf.ug.edu.pl as few different interval representations as possible. In [2] a class of interval graphs whose representations are far from being unique is demonstrated.

Let $\mathcal{I}=\left\{I_{1}, \ldots, I_{p}\right\}$ be a set of intervals of the real line, where $I_{i}=\left[a_{i}, b_{i}\right]$ for $i=1,2, \ldots, p$. An interval $I_{i}$ is called an end interval of the set $\mathcal{I}$ if $a_{i} \leq a_{j}$ for all $j$, or $b_{i} \geq b_{j}$ for all $j$. A graph $G$ is called a homogeneously representable interval graph (shortly, an HRI graph) if for every vertex $v$ of $G$ there exists an interval representation of $G$ in which the interval representing $v$ is an end interval. Homogeneously representable interval graphs were characterized in terms of forbidden subgraphs by Skrien and Gimbel [2].

Theorem 1 (Skrien and Gimbel) A graph $G$ is an HRI graph if and only if it does not contain any of the graphs $P_{4}, C_{4}, C_{5}$ or $G_{1}$ (Fig. 1) as an induced subgraph.

The line graph of a graph $G$, denoted by $L(G)$, is the intersection graph $\Omega(\bar{E}(G))$ of the family $\bar{E}(G)=$ $\{\{u, v\}: u v \in E(G)\}$, that is, $L(G)$ is the graph whose vertices are in one-to-one correspondence with the edges of $G$, and two vertices of $L(G)$ are adjacent if and only if the corresponding edges of $G$ are adjacent. Whitney [3] proved that $K_{1,3}, K_{3}$ is the only pair of non-isomorphic connected graphs with isomorphic line graphs. In the next two theorems we characterize all graphs $G$ whose line graphs $L(G)$ are homogeneously representable interval graphs.

Theorem 2 The line graph $L(G)$ of a graph $G$ is an HRI graph if and only if $G$ contains no $P_{5}, C_{4}, C_{5}$ or $G_{2}$ (Fig. 1) as a subgraph.

Proof Note that $P_{4}=L\left(P_{5}\right), C_{4}=L\left(C_{4}\right), C_{5}=L\left(C_{5}\right)$, and $G_{1}=L\left(G_{2}\right)$. Now, Whitney's theorem implies that if 


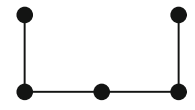

$P_{4}$

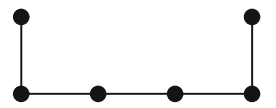

$P_{5}$

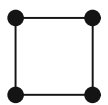

$C_{4}$

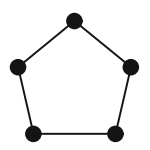

$C_{5}$

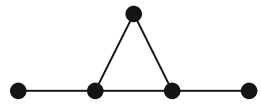

$G_{1}$

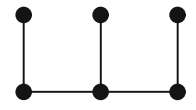

$G_{2}$

Fig. 1 Graphs $P_{4}, P_{5}, C_{4}, C_{5}, G_{1}$ and $G_{2}$

at least one of the graphs $P_{4}, C_{4}, C_{5}$, and $G_{1}$ is an induced subgraph of the line graph $L(G)$, then at least one of the graphs $P_{5}, C_{4}, C_{5}$, and $G_{2}$ is a subgraph of $G$. From this and from Theorem 1 it follows that if $L(G)$ is not an HRI graph, then at least one of the graphs $P_{5}, C_{4}, C_{5}$, and $G_{2}$ is a subgraph of $G$. The opposite implication is straightforward.

Theorem 3 The line graph $L(G)$ of a graph $G$ is an HRI graph if and only if every connected component of $G$ is a subgraph of any of the graphs $H_{1}, H_{2}$, and $H_{3}$ Fig. 2.

Proof Since $L(G)$ is an HRI graph if and only if every connected component of $L(G)$ is an HRI graph, without loss of generality we may assume that $G$ is connected and different from $K_{1}$. First note that if $G$ is a subgraph of any of the graphs given in Fig. 2, then it contains no $P_{5}, C_{4}, C_{5}$ or $G_{2}$ as a subgraph, and therefore $L(G)$ is an HRI graph, by Theorem 2 .

Now assume that $L(G)$ is an HRI graph. According to Theorem 2, the graph $G$ does not contain $P_{5}, C_{4}, C_{5}$ or $G_{2}$ as a subgraph. Let $P=\left(v_{0}, v_{1}, \ldots, v_{d}\right)$ be a longest path in $G$. Since $P_{5}$ is not a subgraph of $G$ and $G \neq K_{1}$, we have $1 \leq d \leq 4$. If $d=1$, then $G=K_{2}$ and $G$ is a subgraph of $H_{i}$. If $d=2$, then $G$ is a star or a complete graph on three vertices. Notice that $G$ is a subgraph of the graphs $H_{1}$ and $H_{2}$. If $d=3$ and $P$ has no chord in $G$, then it follows from the choice of $P$ that the sets $N_{G}\left(v_{1}\right)$ and $N_{G}\left(v_{2}\right)$ are disjoint, and every vertex of $N_{G}\left(v_{1}\right) \cup N_{G}\left(v_{2}\right) \backslash\left\{v_{1}, v_{2}\right\}$ is a leaf in $G$. Thus $G$ is a double star, and it is a subgraph of $H_{2}$. Now assume that $d=3$ and $P$ has a chord in $G$. From the absence of $C_{4}$ in $G$, it follows that either $v_{0} v_{2}$ or $v_{1} v_{3}$ is a chord of $P$ in $G$. Without loss of generality, assume that $v_{0} v_{2}$ is a chord of $P$ in $G$. Since $P$ is a longest path in $G$, we have $N_{G}\left(v_{0}\right)=\left\{v_{1}, v_{2}\right\}, N_{G}\left(v_{1}\right)=\left\{v_{0}, v_{2}\right\}$, and each vertex of $N_{G}\left(v_{2}\right) \backslash\left\{v_{0}, v_{1}\right\}$ is a leaf in $G$. Therefore $G$ can be obtained from $K_{3}$ by attaching a positive number of leaves to exactly one vertex of $K_{3}$. Certainly, $G$ is a subgraph of

Fig. 2 Graphs $H_{1}, H_{2}$ and $H_{3}$

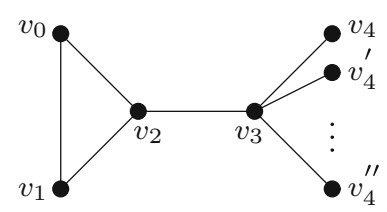

$H_{1}$

$H_{2}$. Now assume that $d=4$. From the absence of $C_{4}$ and $C_{5}$ in $G$ and from the choice of $P$, it easily follows that $N_{G}\left(v_{0}\right) \backslash\left\{v_{1}\right\} \subseteq\left\{v_{2}\right\}$ and $N_{G}\left(v_{4}\right) \backslash\left\{v_{3}\right\} \subseteq\left\{v_{2}\right\}$. In addition, $N_{G}\left(v_{2}\right) \backslash\left\{v_{1}, v_{3}\right\} \subseteq\left\{v_{0}, v_{4}\right\}$ as otherwise $G_{2}$ would be a subgraph of $G$. Again from the choice of $P$ and from the absence of $C_{4}$ in $G$, it follows that $N_{G}\left(v_{1}\right)=\left\{v_{0}, v_{2}\right\}$ if $v_{0} v_{2}$ is a chord of $P$ in $G$. Similarly, $N_{G}\left(v_{3}\right)=\left\{v_{2}, v_{4}\right\}$ if $v_{2} v_{4}$ is a chord of $P$ in $G$. This implies that $G=H_{3}$ if both $v_{0} v_{2}$ and $v_{2} v_{4}$ are chords of $P$ in $G$. If $v_{0} v_{2}$ is a chord of $P$ and $v_{2} v_{4}$ is not a chord of $P$, then the choice of $P$ implies that the vertices belonging to $N_{G}\left(v_{3}\right)$ are independent, and $G$ is a subgraph of $H_{1}$. Similarly, $G$ is a subgraph of $H_{1}$ if $v_{2} v_{4}$ is a chord and $v_{0} v_{2}$ is not a chord of $P$ in $G$. Finally assume that neither $v_{0} v_{2}$ nor $v_{2} v_{4}$ is a chord of $P$ in $G$. Then from the choice of $P$ and from the absence of $C_{4}$ in $G$, it follows that the sets $N_{G}\left(v_{1}\right) \backslash\left\{v_{2}\right\}$ and $N_{G}\left(v_{3}\right) \backslash\left\{v_{2}\right\}$ are disjoint and each of them consists of independent vertices. Therefore $G$ is a subgraph of $\mathrm{H}_{2}$.

The middle graph of a graph $G$, denoted by $M(G)$, is the intersection graph $\Omega(\mathcal{F})$ of the family $\mathcal{F}=\{\{v\}: v \in$ $V(G)\} \cup\{\{v, u\}: v u \in E(G)\}$. It is known that $M(G)$ is isomorphic to the line graph $L\left(G \circ K_{1}\right)$ [4], where $G \circ K_{1}$ is a graph obtained by taking the graph $G$ and $|V(G)|$ copies of $K_{1}$ and then joining the $i$-th vertex of $G$ to the $i$-th copy of $K_{1}$.

The following result follows from Theorems 1 and 2 .

Theorem 4 The middle graph $M(G)$ of a graph $G$ is an HRI graph if and only if every connected component of $G$ is isomorphic to $K_{1}$ or $K_{2}$.

Proof If every component of $G$ is isomorphic to $K_{1}$ or $K_{2}$, then every component of $M(G)$ is $K_{1}=M\left(K_{1}\right)$ or $K_{1,2}=$ $M\left(K_{2}\right)$. Thus by Theorem $1, M(G)$ is an HRI graph. Now assume that $M(G)$ is an HRI graph. Suppose that $G$ has a component different from $K_{1}$ and $K_{2}$. Then $K_{1,2}$ is a

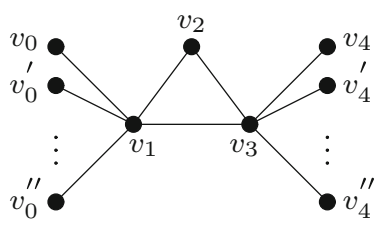

$\mathrm{H}_{2}$

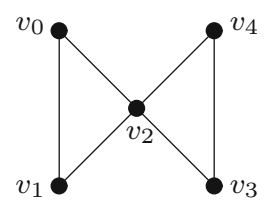

$H_{3}$ 
subgraph of $G$ and therefore $G_{2}=K_{1,2} \circ K_{1}$ is a subgraph of $G \circ K_{1}$. Consequently, by Theorem 2, the middle graph $M(G)=L\left(G \circ K_{1}\right)$ is not an HRI graph, a contradiction.

The total graph of a graph $G$, denoted by $T(G)$, is the intersection graph $\Omega(F)$ of the family $F=\bar{E}(G) \cup$ $\overline{V E}(G)=\{\{v, u\}: v u \in E(G)\} \cup\{\{v\} \cup\{\{v, u\} \quad: u \in$ $\left.\left.N_{G}(v)\right\}: v \in V(G)\right\}$, that is, $T(G)$ is the graph for which there exists a one-to-one correspondence between its vertices and the vertices and edges of $G$ such that two vertices of $T(G)$ are adjacent if and only if the corresponding elements in $G$ are adjacent or incident. This concept was originated by Behzad [5]. It is interesting to note that the graphs $G$ and $L(G)$ are induced subgraphs of the total graph $T(G)$.

We now determine all graphs whose total graphs are HRI graphs.

Theorem 5 The total graph $T(G)$ of a graph $G$ is an HRI graph if and only if every connected component of $G$ is isomorphic to $K_{1}, K_{2}$ or $K_{1,2}$.

Proof The sufficiency follows immediately from Theorem 1. Now assume that $T(G)$ is an HRI graph. It is easy to see that if $T(G)$ is an interval graph, then every connected component of $G$ is triangle-free. From this and from the absence of $G_{1}$ in $T(G)$ (Theorem 1) it follows that $P_{3}$ is not a subgraph of $G$. Thus every component of $G$ is isomorphic to one of the graphs $K_{1}, K_{2}$, or $K_{1,2}$.

Acknowledgments Research partially supported by the Polish National Science Centre Grant 2011/02/A/ST6/00201.

Open Access This article is distributed under the terms of the Creative Commons Attribution License which permits any use, distribution, and reproduction in any medium, provided the original author(s) and the source are credited.

\section{References}

1. Lekkerkerker C, Boland J (1962) Representation of a finite graph by a set of intervals on the real line. Fund Math 51:45-64

2. Skrien D, Gimbel J (1985) Homogeneously representable interval graphs. Discret Math 55:213-216

3. Whitney H (1932) Congruent graphs and the connectivity of graphs. Am J Math 54:150-168

4. Akiyama J, Hamada T, Yoshimura I (1974) Miscellaneous properties of middle graphs. TRU Math 10:41-53

5. Behzad M (1967) A criterion for the planarity of the total graph of a graph. Math Proc Cambridge Philos Soc 63:679-681 